\title{
Synthesis of the isotopes of elements 118 and 116 in the ${ }^{249} \mathrm{Cf}$ and ${ }^{245} \mathrm{Cm}+{ }^{48} \mathrm{Ca}$ fusion reactions
}

Yu. Ts. Oganessian, V. K. Utyonkov, Yu. V. Lobanov, F. Sh. Abdullin, A. N. Polyakov, R. N. Sagaidak, I. V. Shirokovsky, Yu. S. Tsyganov, A. A. Voinov, G. G. Gulbekian, S. L. Bogomolov, B. N. Gikal, A. N. Mezentsev, S. Iliev, V. G. Subbotin, A. M. Sukhov, K. Subotic, V. I. Zagrebaev, G. K. Vostokin, and M. G. Itkis Joint Institute for Nuclear Research, 141980 Dubna, Russian Federation

\author{
K. J. Moody, J. B. Patin, D. A. Shaughnessy, M. A. Stoyer, N. J. Stoyer, P. A. Wilk, J. M. Kenneally, \\ J. H. Landrum, J. F. Wild, and R. W. Lougheed \\ University of California, Lawrence Livermore National Laboratory, Livermore, California 94551, USA \\ (Received 31 January 2006; revised manuscript received 23 August 2006; published 9 October 2006)
}

\begin{abstract}
The decay properties of ${ }^{290} 116$ and ${ }^{291} 116$, and the dependence of their production cross sections on the excitation energies of the compound nucleus, ${ }^{293} 116$, have been measured in the ${ }^{245} \mathrm{Cm}\left({ }^{48} \mathrm{Ca}, x n\right){ }^{293-x} 116$ reaction. These isotopes of element 116 are the decay daughters of element 118 isotopes, which are produced via the ${ }^{249} \mathrm{Cf}+{ }^{48} \mathrm{Ca}$ reaction. We performed the element 118 experiment at two projectile energies, corresponding to ${ }^{297} 118$ compound nucleus excitation energies of $E^{*}=29.2 \pm 2.5$ and $34.4 \pm 2.3 \mathrm{MeV}$. During an irradiation with a total beam dose of $4.1 \times 10^{19}{ }^{48} \mathrm{Ca}$ projectiles, three similar decay chains consisting of two or three consecutive $\alpha$ decays and terminated by a spontaneous fission (SF) with high total kinetic energy of about $230 \mathrm{MeV}$ were observed. The three decay chains originated from the even-even isotope ${ }^{294} 118\left(E_{\alpha}=11.65 \pm\right.$ $\left.0.06 \mathrm{MeV}, T_{\alpha}=0.89_{-0.31}^{+1.07} \mathrm{~ms}\right)$ produced in the $3 n$-evaporation channel of the ${ }^{249} \mathrm{Cf}+{ }^{48} \mathrm{Ca}$ reaction with a maximum cross section of $0.5_{-0.3}^{+1.6} \mathrm{pb}$.
\end{abstract}

DOI: 10.1103/PhysRevC.74.044602 PACS number(s): 25.70.Gh, 23.60.+e, 25.85.Ca, 27.90.+b

\section{INTRODUCTION}

The existence of an enhanced stability in the region of the superheavy nuclei, which has been developed in various theoretical approaches and hypothesized for about 40 years, has been validated by recent experiments. Decay energies and lifetimes of 28 new nuclides with $Z=104-116$ and $N=$ 162-177 that have been synthesized in the complete-fusion reactions of ${ }^{238} \mathrm{U},{ }^{242,244} \mathrm{Pu},{ }^{243} \mathrm{Am}$, and ${ }^{245,248} \mathrm{Cm}$ targets with ${ }^{48} \mathrm{Ca}$ beams indicate a considerable increase of the stability of superheavy nuclei with an increasing number of neutrons [1-3]. As a whole, the results of the experiments agree with the predictions of theoretical models concerning the properties of the superheavy nuclei in the vicinity of closed nuclear shells.

Practically all of the model calculations predict the existence of a closed spherical shell at $N=184$; however, they differ in predicting the atomic number of the closed proton shell. At the same time, the boundaries of the "Island of Stability" are rather sensitive to the parameters of these models; most importantly the magnitude of the shell effect for the heaviest nuclei substantially depends on the magic proton number $Z_{\text {shell }}$. The difference between the models increases when the decay properties of the isotopes of elements beyond $Z=116$ are predicted.

For instance, according to the macroscopic-microscopic model $(\mathrm{MM})$ with $Z_{\text {shell }}=114$ [4,5], the even-even nucleus with $Z=118$ and $A=294$ would undergo $\alpha$ decay with an energy $Q_{\alpha}=11.9-12.1 \mathrm{MeV}$ and a half-life $T_{\alpha} \approx 0.1 \mathrm{~ms}$. In the purely microscopic Hartree-Fock-Bogoliubov (HFB) model, with $Z_{\text {shell }}=124,126$ [6-10], the values $Q_{\alpha}=11.2-$ $11.6 \mathrm{MeV}$ and $T_{\alpha} \approx 1-10 \mathrm{~ms}$ are predicted. The relativistic mean-field $(\mathrm{RMF})$ calculations with $Z_{\text {shell }}=120 \quad[11,12]$ give $Q_{\alpha}=11.0-11.2 \mathrm{MeV}$ and $T_{\alpha} \approx 10-50 \mathrm{~ms}$. The decay energies arising in the three calculations covers more than $1 \mathrm{MeV}$ and, accordingly, the half-life value $T_{\alpha}$ varies by more than two orders of magnitude.

The differences between fission barrier heights that are predicted by various models for the isotopes of element 118 would also significantly influence the survivability of the compound nucleus and, accordingly, the cross section for the production of evaporation residues (ER). Because lower fission barriers are predicted in the MM model, the expected formation cross sections of nuclei with $Z=118$ should be lower than those of the isotopes of element 114, while the microscopic models with the proton shell at $Z \geqslant 120$ predict higher fission barriers for $Z=118$ nuclei which might, on the contrary, result in larger cross sections for $x n$-evaporation channels.

The first attempt to synthesize element 118 was undertaken in 1999 using the ${ }^{208} \mathrm{~Pb}\left({ }^{86} \mathrm{Kr}, n\right){ }^{293} 118$ cold fusion reaction. The theoretically expected high fusion-evaporation cross section of the reaction with the magic ${ }^{86} \mathrm{Kr}$ projectile [13] was later disproved and several experiments yielded a cross section limit of $\sigma_{n} \leqslant 0.2 \mathrm{pb}$ [14-17].

In 2002, we attempted to produce element 118 in the ${ }^{249} \mathrm{Cf}+{ }^{48} \mathrm{Ca}$ reaction at a ${ }^{297} 118$ compound nucleus excitation energy of $E^{*}=29 \mathrm{MeV}$, which was formed essentially at the Coulomb barrier of the reaction. In a continuous 2300-h experiment, with an accumulated beam dose of $2.5 \times$ $10^{19}$ particles, we detected a single decay chain of correlated decays $(\alpha-\alpha-\mathrm{SF})$ with decay energies and times close to those expected for the even-even isotope ${ }^{294} 118$ - the product of the $3 n$-evaporation channel of the ${ }^{249} \mathrm{Cf}\left({ }^{48} \mathrm{Ca}, x n\right){ }^{297-x} 118$ reaction [18]. During the next two years, using the ${ }^{238} \mathrm{U}$, ${ }^{242,244} \mathrm{Pu}$ and ${ }^{245,248} \mathrm{Cm}+{ }^{48} \mathrm{Ca}$ reactions, we performed a series of experiments to synthesize and determine the decay 
properties of a number of isotopes of elements 112,114 and 116 , including the even-even nuclides ${ }^{282} 112,{ }^{286} 114$ and ${ }^{290} 116$ - the members of the ${ }^{294} 118$ decay chain [1,2]. The properties of the nuclei produced in these experiments corroborated the assignment of the first observed event to the decay of ${ }^{294} 118$.

In the present work, the investigation of the synthesis of element 118 was continued. In February-March and MayJune, 2005, we carried out two experimental runs with the ${ }^{245} \mathrm{Cm}+{ }^{48} \mathrm{Ca}$ and ${ }^{249} \mathrm{Cf}+{ }^{48} \mathrm{Ca}$ reactions. These two experiments were motivated by the fact that the compound nuclei ${ }^{293} 116$ and ${ }^{297} 118$ formed in these reactions differ in charge and mass by a single $\alpha$ particle. At the Coulomb barriers [19] of the reactions, the compound nuclei are characterized by similar excitation energies of 28.9 and $26.6 \mathrm{MeV}$, respectively. In the ${ }^{245} \mathrm{Cm}\left({ }^{48} \mathrm{Ca}, x n\right){ }^{293-x} 116$ reaction, in addition to previously reported data [1], two isotopes, ${ }^{290} 116$ and ${ }^{291} 116$, were identified independently through the measurement of excitation functions for the $2 n$ - and $3 n$-evaporation channels in the excitation energy range $E^{*}=38-43 \mathrm{MeV}$. From the results of the ${ }^{245} \mathrm{Cm}$ run, we could thus choose the optimal experimental conditions for synthesizing isotopes of element 118 in the ${ }^{249} \mathrm{Cf}+{ }^{48} \mathrm{Ca}$ reaction. Thus, the $\alpha$ decay of the $Z=118$ nuclides should produce the previously characterized element 116 nuclides as well as the decay chains observed in the same evaporation channels from the ${ }^{245} \mathrm{Cm}+{ }^{48} \mathrm{Ca}$ reaction.

\section{EXPERIMENTAL TECHNIQUE}

The experimental set up is analogous to that used in our previous experiments [1-3]. The ${ }^{48} \mathrm{Ca}$-ion beam was accelerated by the U400 cyclotron of the Flerov Laboratory of Nuclear Reactions, Joint Institute for Nuclear Research in Dubna. The typical beam intensity at the target was $1.2 \mathrm{p} \mu \mathrm{A}$. The beam energy was determined and controlled by employing a time-of-flight system with a systematic uncertainty of $1 \mathrm{MeV}$.

The $32-\mathrm{cm}^{2}$ rotating targets consisted of the enriched isotopes ${ }^{249} \mathrm{Cf}(>98 \%)$ and ${ }^{245} \mathrm{Cm}(98.7 \%)$ deposited as oxides onto $1.5-\mu \mathrm{m}$ Ti foils. The integrity of the target layers was checked periodically by measuring the ${ }^{249} \mathrm{Cf}$ and ${ }^{245} \mathrm{Cm}$ $\alpha$-particle counting rate.

The evaporation residues recoiling from the target were separated in flight from ${ }^{48} \mathrm{Ca}$ beam ions, scattered particles and transfer-reaction products by the Dubna gas-filled recoil separator [20]. The transmission efficiency of the separator for $Z=116$ and 118 nuclei is estimated to be approximately $35 \%$ [20]. ERs passed through a time-of-flight system (TOF) (with detection efficiency of $99.9 \%$ ) and were implanted in a $4-\mathrm{cm} \times 12-\mathrm{cm}$ semiconductor detector array with 12 vertical position-sensitive strips surrounded by eight $4-\mathrm{cm} \times 4-\mathrm{cm}$ side detectors without position sensitivity, forming a box open to the front (beam) side. The position-averaged detection efficiency for full-energy $\alpha$ particles emitted in the decays of implanted nuclei was $87 \%$.

The detection system was tested by registering the recoil nuclei and decays ( $\alpha$ or SF) of known isotopes of No and $\mathrm{Th}$, as well as their descendants, produced in the reactions ${ }^{206} \mathrm{~Pb}\left({ }^{48} \mathrm{Ca}, \mathrm{xn}\right)$ and ${ }^{\text {nat }} \mathrm{Yb}\left({ }^{48} \mathrm{Ca}, \mathrm{xn}\right)$, respectively. The energy resolutions were $70-120 \mathrm{keV}$ (depending on strip) for $\alpha$ particles absorbed in the focal-plane detector, 280 $410 \mathrm{keV}$ for $\alpha$ particles that escaped this detector with a low energy release and registered by a side detector, and $0.5 \mathrm{MeV}$ for $\alpha$ particles detected only by a side detector (without a focal-plane position signal). Fission fragments from the decay of ${ }^{252} \mathrm{No}$ implants produced in the ${ }^{206} \mathrm{~Pb}+{ }^{48} \mathrm{Ca}$ reaction were used for the total kinetic energy (TKE) calibration. The measured fragment energies presented in this work were not corrected for the pulse-height defect of the detectors or for energy losses of escaping fragments in the detectors and the pentane gas filling the detection system. The mean sum energy loss of fission fragments emitted in the SF decay of ${ }^{252} \mathrm{No}$ was about $20 \mathrm{MeV}$. From the data of previously registered $\mathrm{SF}$ nuclei in ${ }^{48} \mathrm{Ca}$-induced reactions (see Refs. [1-3] and references therein) it follows that the signals of SF fragments of nuclei heavier than ${ }^{252}$ No are expected to have energies $E_{F 1} \geqslant 130 \mathrm{MeV}$ for SF events absorbed only in the focal-plane detector and $E_{\mathrm{F} 1}+E_{\mathrm{F} 2} \geqslant 170 \mathrm{MeV}$ for fragments registered by both detectors. For most of the strips, the FWHM position resolutions of the signals of correlated decays of nuclei implanted in the detectors were $0.8-1.3 \mathrm{~mm}$ for ER- $\alpha$ signals and $0.4-0.8 \mathrm{~mm}$ for ER-SF signals. If an $\alpha$ particle was detected by both the focal-plane and a side detector, the position resolution depended on the amplitude of the signal in the focal-plane detector (see, e.g., Fig. 4 in Ref. [18]), but was generally inferior to that obtained for the full-energy signal.

The experimental conditions are summarized in Table I. Excitation energies of the compound nuclei at given projectile energies are calculated using the masses of Ref. [21], taking into account the thickness of the targets and the energy spread of the incident cyclotron beam. The beam energy losses in the separator's entrance window and target backing (both $1.5-\mu \mathrm{m}$ Ti foils) and target layer were calculated using available data of Hubert et al. or Northcliffe and Schilling in other cases [22].

For detection of expected sequential decays of the daughter nuclides in the absence of beam-associated background, the beam was switched off after a recoil signal was detected with parameters of implantation energy $E_{\mathrm{ER}}=7-16 \mathrm{MeV}$ expected for complete-fusion evaporation residues, followed by an $\alpha$-like signal with an energy of $9.9 \mathrm{MeV} \leqslant E_{\alpha 1} \leqslant$ $12.0 \mathrm{MeV}$ or $9.9 \mathrm{MeV} \leqslant E_{\alpha 1} \leqslant 11.3 \mathrm{MeV}$ for $Z=118$ and 116 recoils, respectively, in the same strip, within a 1.8-2.5-mm wide position window and a time interval of

TABLE I. Target thicknesses corresponding to isotope quantity, reaction-specific lab-frame beam energies in the middle of the target layers, excitation energy intervals and total beam doses for the given reactions.

\begin{tabular}{lccccc}
\hline \hline Reaction & $\begin{array}{c}\text { Target } \\
\text { thickness } \\
\left(\mathrm{mg} / \mathrm{cm}^{2}\right)\end{array}$ & $\begin{array}{c}E_{\text {beam }} \\
(\mathrm{MeV})\end{array}$ & $\begin{array}{c}E^{*} \\
(\mathrm{MeV})\end{array}$ & Beam dose & Reference \\
\hline${ }^{249} \mathrm{Cf}+{ }^{48} \mathrm{Ca}$ & 0.23 & 245 & $26.6-31.7$ & $2.5 \times 10^{19}$ & {$[18]$} \\
& 0.34 & 251 & $32.1-36.6$ & $1.6 \times 10^{19}$ & this work \\
\hline${ }^{245} \mathrm{Cm}+{ }^{48} \mathrm{Ca}$ & 0.35 & 243 & $30.9-35.0$ & $1.2 \times 10^{19}$ & {$[1]$} \\
& 0.34 & 249 & $35.9-39.9$ & $5.4 \times 10^{18}$ & this work \\
& 0.34 & 255 & $40.7-44.8$ & $8.3 \times 10^{18}$ & this work \\
\hline \hline
\end{tabular}


$\Delta t \leqslant 1$ s. If, during the first 1-min beam-off time interval, an $\alpha$ particle with $E_{\alpha 2}=9.5-11.15 \mathrm{MeV}$ was registered in any position of the same strip, the beam-off interval was automatically extended to $12 \mathrm{~min}$.

\section{EXPERIMENTAL RESULTS AND DISCUSSION}

\section{A. Experiments with a ${ }^{245} \mathrm{Cm}$ target}

In two series of ${ }^{245} \mathrm{Cm}+{ }^{48} \mathrm{Ca}$ irradiations, approximately 1990 beam-off intervals occurred, for a total of $33 \mathrm{~h}$. The spectrum of $\alpha$-like signals (all events without a registered TOF signal) in all strips in the energy range $7 \leqslant E_{\alpha} \leqslant$ $12 \mathrm{MeV}$ accumulated over the whole 1000 -hour ${ }^{245} \mathrm{Cm}+{ }^{48} \mathrm{Ca}$ experiment is shown in Fig. 1(a). This figure also shows the $\alpha$-particle spectrum detected during beam-off time intervals. In the high-energy part of the $\alpha$-particle spectrum, where the decays of daughter nuclei of isotopes ${ }^{289-291} 116\left(E_{\alpha}=\right.$ 9.5-10.5 MeV) are expected, only 11 events were detected. Two of them (shown by arrows), as we will demonstrate in the following discussion, belong to the decay of ${ }^{286} 114$, the daughter isotope of ${ }^{290} 116$.

The total spectrum of high-energy signals with $E \geqslant 50 \mathrm{MeV}$ (without an associated TOF signal) is presented in Fig. 1(b). In cases when fission signals were registered by both the focalplane and the side detector, the sum energy is given. In the ${ }^{245} \mathrm{Cm}+{ }^{48} \mathrm{Ca}$ reaction, of the 24 signals detected with energies expected for fission of heavy nuclei, nine are assigned to the decays of descendant nuclei of the $Z=116$ isotopes. The other signals with energy $E \leqslant 130 \mathrm{MeV}$ could be explained in part by scattered ${ }^{48} \mathrm{Ca}$ and projectile-like particles, as well as by scattered fragments of the induced fission of the target (they are not observed in the beam-off spectrum). The background SF events with energy $E \geqslant 130 \mathrm{MeV}$ can be caused by longlived nuclei produced in transfer reactions during previous experiments where the same set of detectors was used. Some of these events were observed during beam-off periods. However, for these 15 events no ER-SF correlations were found.
The measured parameters of the members of the decay chains observed in the ${ }^{245} \mathrm{Cm}$ irradiations are presented in the second part of Table II. In these experiments, we registered nine new decay chains of ${ }^{290} 116$ : five chains at the ${ }^{48} \mathrm{Ca}$ energy $E_{\text {lab }}=249 \mathrm{MeV}$, and four decays at the higher beam energy $E_{\text {lab }}=255 \mathrm{MeV}$. We postulate that in three of the decay chains, the $\alpha$ particles arising from the decay of ${ }^{290} 116$ escaped the detector array with signals in the focal-plane detector below the threshold $(\approx 1 \mathrm{MeV})$. In Table II, such events are marked "Missing $\alpha$." Three missing $\alpha$ particles out of $23 \alpha$ decays observed during both experiments is entirely consistent with the $87 \%$-efficiency for the detection of $\alpha$ particles by our detector array. One "missing $\alpha$ " occurred at $E_{\mathrm{lab}}=249 \mathrm{MeV}$ and two others at $E_{\mathrm{lab}}=255 \mathrm{MeV}$. In the first case, the location of the missing $\alpha$ within the decay chain of type ER- $\alpha_{1}-\alpha_{2}$-SF can be easily determined by comparison with the other four chains, and the energies and decay times of the observed $\alpha$ particle and SF decay; $E_{\alpha 2}=$ $10.22 \mathrm{MeV}, \delta t_{\alpha 2}=62.9 \mathrm{~ms}$ and $E_{\mathrm{SF}}=209 \mathrm{MeV}, \delta t_{\mathrm{SF}}=$ $0.746 \mathrm{~ms}$, respectively. In the other two cases where ER-SF chains were observed, the probability of losing two $\alpha$ particles in each decay chain is less than $2 \%$ and the probability of random ER-SF correlations is less than $1 \%$. Taking into account registration times $\delta t_{\mathrm{SF}}=876.5 \mathrm{~ms}$ and $252.3 \mathrm{~ms}$, which are comparable with the lifetime of the daughter isotope ${ }^{286} 114$, and $\sim 50 \%$ probability of spontaneous fission for ${ }^{286} 114$ observed in previous experiments [2], we assign the SF to the daughter isotope ${ }^{286} 114$, assuming that in both of these cases the $\alpha$ particles of ${ }^{290} 116$ were not registered.

For all observed decay chains, the position deviations of the detected signals of $Z=116$ recoiling nuclei and subsequent sequential decays ( $\alpha$ and SF) are consistent with position resolutions of that particular strip detector (see Table II). The correlated positions coupled with short decay times indicate true, nonrandom correlations between registered events. Only two SF signals, with position deviations of 5.1 and $3.4 \mathrm{~mm}$, exceed the ER-SF position resolution for those strips.
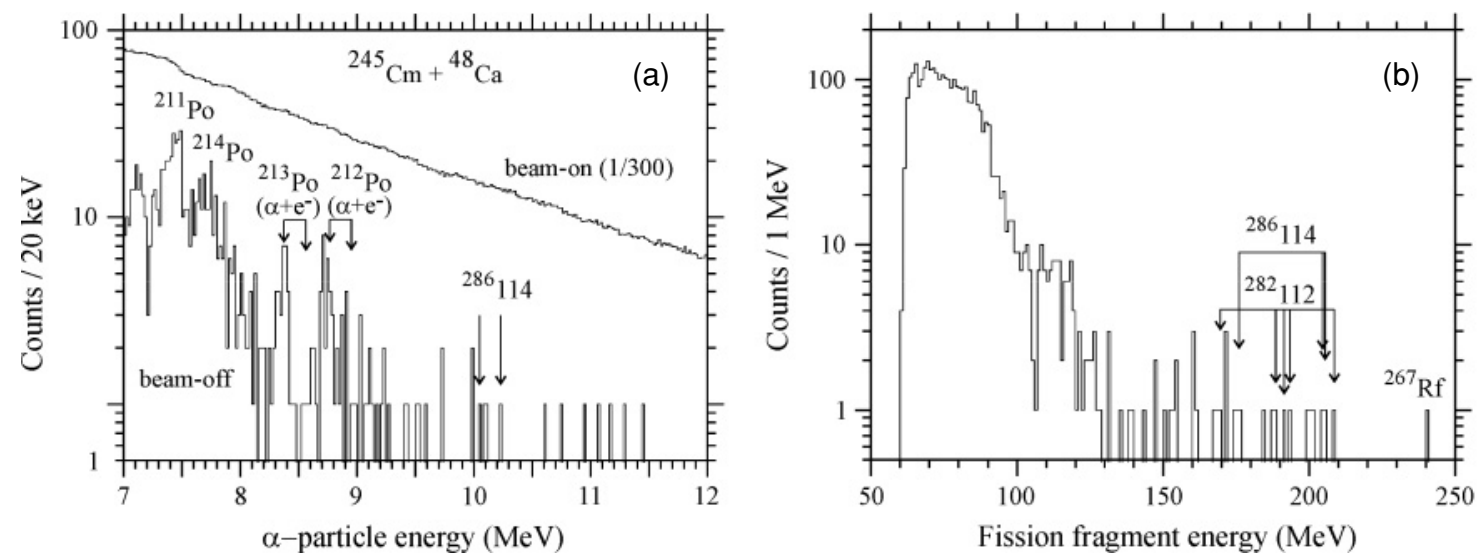

FIG. 1. (a) Total beam-on and beam-off $\alpha$-particle energy spectra of events registered by the focal-plane detector and by both the focal-plane and side detectors in the ${ }^{245} \mathrm{Cm}+{ }^{48} \mathrm{Ca}$ reaction. In the beam-off $\alpha$-particle spectrum we observe the peaks originating from isotopes of Po, the decay products of long-lived isotopes of Ra-Th produced in transfer reactions, and ${ }^{211} \mathrm{Po}$, the descendant nucleus of ${ }^{219} \mathrm{Th}$ produced in the calibrations with $a^{\text {nat }} \mathrm{Yb}$ target. The energies of events observed during beam-off periods in the correlated decay chains are shown by arrows (see Table II). (b) Total fission-fragment energy spectrum in the ${ }^{245} \mathrm{Cm}+{ }^{48} \mathrm{Ca}$ reaction; the arrows show the energies of events observed in the correlated decay chains. 
TABLE II. Observed decay chains. The first four columns show detector strip numbers, lab-frame beam energies in the middle of the target layers, ER energies, and ER positions with respect to the top of the strips. For the following decays the time intervals between events $(\delta \mathrm{t}), \alpha$-particle and SF fragment energies (E), and the differences in vertical positions relative to the ER $(\delta \mathrm{P})$ are shown. Bold events were registered during a beam-off period. The $\alpha$-particle energy errors are shown in parentheses. Time intervals for events following a "missing $\alpha$ " were measured from preceding registered events and are shown in italic.

\begin{tabular}{|c|c|c|c|c|c|c|c|c|c|c|c|c|c|c|c|}
\hline \multicolumn{16}{|c|}{${ }^{249} \mathrm{Cf}+{ }^{48} \mathrm{Ca}$} \\
\hline \multirow[t]{2}{*}{$\overline{\text { Str }}$} & \multirow[t]{2}{*}{$\mathrm{E}_{\mathrm{L}}(\mathrm{MeV})$} & \multicolumn{2}{|c|}{${ }^{294} 118$} & \multicolumn{3}{|c|}{${ }^{294} 118$} & \multicolumn{3}{|c|}{${ }^{290} 116$} & \multicolumn{3}{|c|}{${ }^{286} 114$} & \multicolumn{3}{|c|}{${ }^{282} 112$} \\
\hline & & $\begin{array}{c}E_{E R} \\
(\mathrm{MeV})\end{array}$ & $\begin{array}{c}\mathrm{P}_{\mathrm{ER}} \\
(\mathrm{mm})\end{array}$ & $\begin{array}{c}\delta \mathrm{t}_{\mathrm{ER}-\alpha} \\
(\mathrm{ms})\end{array}$ & $\begin{array}{c}\mathrm{E}_{\alpha} \\
(\mathrm{MeV})\end{array}$ & $\begin{array}{c}\delta \mathrm{P}_{\mathrm{ER}-\alpha} \\
(\mathrm{mm})\end{array}$ & $\begin{array}{l}\delta \mathrm{t}_{\alpha-\alpha} \\
(\mathrm{ms})\end{array}$ & $\begin{array}{c}\mathrm{E}_{\alpha} \\
(\mathrm{MeV})\end{array}$ & $\begin{array}{c}\delta \mathrm{P}_{\mathrm{ER}-\alpha} \\
(\mathrm{mm})\end{array}$ & $\begin{array}{c}\delta \mathrm{t}_{\alpha-\alpha / \mathrm{SF}} \\
(\mathrm{ms})\end{array}$ & $\begin{array}{l}\mathrm{E}_{\alpha / \mathrm{SF}} \\
(\mathrm{MeV})\end{array}$ & $\begin{array}{c}\delta \mathrm{P}_{\mathrm{ER}-\alpha / \mathrm{SF}} \\
(\mathrm{mm})\end{array}$ & $\begin{array}{c}\delta \mathrm{t}_{\alpha-\mathrm{SF}} \\
(\mathrm{ms})\end{array}$ & $\begin{array}{c}E_{\mathrm{SF}} \\
(\mathrm{MeV})\end{array}$ & $\begin{array}{c}\delta \mathrm{P}_{\mathrm{ER}-\mathrm{SF}} \\
(\mathrm{mm})\end{array}$ \\
\hline 3 & 245 & 13.2 & 8.9 & 2.549 & $11.65(6)$ & -0.1 & 42.1 & $10.71(17)^{b}$ & -6.2 & 517.6 & $207^{\mathrm{e}}$ & -0.5 & & & \\
\hline $7^{\mathrm{a}}$ & 251 & 10.4 & 27.1 & 0.465 & $11.65(10)$ & -0.7 & 1.012 & $10.84(10)$ & -0.7 & 11.39 & 157 & -2.5 & & & \\
\hline 1 & 251 & 13.7 & 17.5 & 0.847 & $11.8(5)^{\mathrm{c}}$ & & 0.098 & $10.80(9)$ & +0.5 & 153.0 & $10.16(9)$ & +0.7 & 2.70 & $202^{\mathrm{e}}$ & +0.6 \\
\hline \multicolumn{16}{|c|}{${ }^{245} \mathrm{Cm}+{ }^{48} \mathrm{Ca}$} \\
\hline \multirow[t]{2}{*}{$\overline{\mathrm{St}}$} & $\mathrm{E}_{\mathrm{L}}(\mathrm{MeV})$ & \multicolumn{2}{|c|}{${ }^{291} 116$} & \multicolumn{3}{|c|}{${ }^{291} 116$} & \multicolumn{3}{|c|}{${ }^{287} 114$} & \multicolumn{3}{|c|}{${ }^{283} 112$} & \multicolumn{3}{|c|}{${ }^{279}$ Ds } \\
\hline & & $\begin{array}{c}E_{\mathrm{ER}} \\
(\mathrm{MeV})\end{array}$ & $\begin{array}{c}\mathrm{P}_{\mathrm{ER}} \\
(\mathrm{mm})\end{array}$ & $\begin{array}{c}\delta \mathrm{t}_{\mathrm{ER}-\alpha} \\
(\mathrm{ms})\end{array}$ & $\begin{array}{c}\mathrm{E}_{\alpha} \\
(\mathrm{MeV})\end{array}$ & $\begin{array}{c}\delta \mathrm{P}_{\mathrm{ER}-\alpha} \\
(\mathrm{mm})\end{array}$ & $\begin{array}{l}\delta \mathrm{t}_{\alpha-\alpha} \\
(\mathrm{ms})\end{array}$ & $\begin{array}{c}\mathrm{E}_{\alpha} \\
(\mathrm{MeV})\end{array}$ & $\begin{array}{c}\delta \mathrm{P}_{\mathrm{ER}-\alpha} \\
(\mathrm{mm})\end{array}$ & $\begin{array}{l}\delta \mathrm{t}_{\alpha-\alpha} \\
(\mathrm{ms})\end{array}$ & $\begin{array}{c}\mathrm{E}_{\alpha} \\
(\mathrm{MeV})\end{array}$ & $\begin{array}{c}\delta \mathrm{P}_{\mathrm{ER}-\alpha} \\
(\mathrm{mm})\end{array}$ & $\begin{array}{c}\delta \mathrm{t}_{\alpha-\alpha} \\
(\mathrm{s})\end{array}$ & $\begin{array}{c}\mathrm{E}_{\alpha} \\
(\mathrm{MeV})\end{array}$ & $\begin{array}{l}\delta \mathrm{P}_{\mathrm{ER}-\alpha} \\
(\mathrm{mm})\end{array}$ \\
\hline \multirow[t]{4}{*}{5} & 249 & 8.6 & 29.7 & 61.1 & $10.76(12)$ & +0.6 & 9.430 & $10.01(12)$ & +0.5 & 34.6 & $9.57(12)$ & +0.5 & 0.707 & $9.70(12)$ & +0.3 \\
\hline & & & & \multicolumn{3}{|c|}{${ }^{275} \mathrm{Hs}$} & \multicolumn{3}{|c|}{${ }^{271} \mathrm{Sg}$} & \multicolumn{3}{|c|}{${ }^{267} \mathrm{Rf}$} & & & \\
\hline & & & & $\begin{array}{c}\delta \mathrm{t}_{\alpha-\alpha} \\
(\mathrm{s})\end{array}$ & $\begin{array}{c}\mathrm{E}_{\alpha} \\
(\mathrm{MeV})\end{array}$ & $\begin{array}{c}\delta \mathrm{P}_{\mathrm{ER}-\alpha} \\
(\mathrm{mm})\end{array}$ & $\begin{array}{c}\delta \mathrm{t}_{\alpha-\alpha} \\
(\mathrm{s})\end{array}$ & $\begin{array}{c}\mathrm{E}_{\alpha} \\
(\mathrm{MeV})\end{array}$ & $\begin{array}{c}\delta \mathrm{P}_{\mathrm{ER}-\alpha} \\
(\mathrm{mm})\end{array}$ & $\begin{array}{l}\delta \mathrm{t}_{\alpha-\mathrm{SF}} \\
(\mathrm{min})\end{array}$ & $\begin{array}{c}E_{\mathrm{SF}} \\
(\mathrm{MeV})\end{array}$ & $\begin{array}{c}\delta \mathrm{P}_{\mathrm{ER}-\mathrm{SF}} \\
(\mathrm{mm})\end{array}$ & & & \\
\hline & & & & 0.3679 & $9.56(36)^{d}$ & & 94.10 & $8.85(36)^{\mathrm{b}}$ & -1.2 & 20.998 & $240^{\mathrm{e}}$ & +0.6 & & & \\
\hline \multirow[t]{2}{*}{ Str } & $\mathrm{E}_{\mathrm{L}}(\mathrm{MeV})$ & \multicolumn{2}{|c|}{${ }^{290} 116$} & \multicolumn{3}{|c|}{${ }^{290} 116$} & \multicolumn{3}{|c|}{${ }^{286} 114$} & \multicolumn{3}{|c|}{${ }^{282} 112$} & & & \\
\hline & & $\begin{array}{c}\mathrm{E}_{\mathrm{ER}} \\
(\mathrm{MeV})\end{array}$ & $\begin{array}{c}\mathrm{P}_{\mathrm{ER}} \\
(\mathrm{mm})\end{array}$ & $\begin{array}{c}\delta \mathrm{t}_{\mathrm{ER}-\alpha} \\
(\mathrm{ms})\end{array}$ & $\begin{array}{c}\mathrm{E}_{\alpha} \\
(\mathrm{MeV})\end{array}$ & $\begin{array}{c}\delta \mathrm{P}_{\mathrm{ER}-\alpha} \\
(\mathrm{mm})\end{array}$ & $\begin{array}{c}\delta \mathrm{t}_{\alpha-\alpha / \mathrm{SF}} \\
(\mathrm{ms})\end{array}$ & $\begin{array}{l}\mathrm{E}_{\alpha / \mathrm{SF}} \\
(\mathrm{MeV})\end{array}$ & $\begin{array}{c}\delta \mathrm{P}_{\mathrm{ER}-\alpha / \mathrm{SF}} \\
(\mathrm{mm})\end{array}$ & $\begin{array}{c}\delta \mathrm{t}_{\alpha-\mathrm{SF}} \\
(\mathrm{ms})\end{array}$ & $\begin{array}{c}\mathrm{E}_{\mathrm{SF}} \\
(\mathrm{MeV})\end{array}$ & $\begin{array}{c}\delta \mathrm{P}_{\mathrm{ER}-\mathrm{SF}} \\
(\mathrm{mm})\end{array}$ & & & \\
\hline 4 & 249 & 11.7 & 25.8 & 0.207 & $11.15(31)^{d}$ & & 107.8 & $10.08(31)^{b}$ & -3.6 & 1.929 & 170 & -0.9 & & & \\
\hline 5 & 249 & 10.2 & 24.7 & 20.1 & $10.81(12)$ & +0.3 & 54.1 & $10.05(12)$ & +0.3 & 1.636 & 188 & +5.1 & & & \\
\hline 4 & 249 & 13.3 & 31.6 & 10.0 & $10.8(5)^{\mathrm{c}}$ & & 137.9 & $10.19(11)$ & -0.3 & 1.020 & 192 & -0.3 & & & \\
\hline $8^{a}$ & 249 & 12.7 & 24.1 & 5.251 & $10.89(8)$ & -0.4 & 40.3 & $10.2(5)^{\mathrm{c}}$ & & 2.423 & 193 & +1.7 & & & \\
\hline 1 & 249 & 11.3 & 36.1 & & Missing $\alpha$ & & 62.9 & $10.22(11)$ & +0.5 & 0.746 & $209^{\mathrm{e}}$ & +0.4 & & & \\
\hline 2 & 255 & 11.2 & 23.6 & & Missing $\alpha$ & & 876.5 & $176^{\mathrm{e}}$ & 0.0 & & & & & & \\
\hline 1 & 255 & 11.7 & 23.5 & 0.792 & $10.87(11)$ & +0.3 & 339.3 & $10.23(11)$ & 0.0 & & Missing SF & & & & \\
\hline $11^{\mathrm{a}}$ & 255 & 8.4 & 19.1 & & Missing $\alpha$ & & 252.3 & $203^{\mathrm{e}}$ & -0.2 & & & & & & \\
\hline $6^{\mathrm{a}}$ & 255 & 14.7 & 19.2 & 22.29 & $10.6(5)^{\mathrm{c}}$ & & 126.4 & $205^{\mathrm{e}}$ & +3.4 & & & & & & \\
\hline
\end{tabular}

The FWHM position resolutions of the signals of correlated decays of nuclei implanted in these strips were 1.9-3.0 mm for ER- $\alpha$ signals and 1.1-2.2 mm for ER-SF signals.

${ }^{\mathrm{b}} \alpha$ particle registered by both focal-plane and side detectors.

${ }^{\mathrm{c}}$ Escaped $\alpha$ particle registered by side detector only.

${ }^{\mathrm{d}}$ Escaped $\alpha$ particle registered by focal-plane detector without position signal because of low deposited energy.

${ }^{\mathrm{e}}$ Fission event registered by both focal-plane and side detectors.

However, one of them was detected during a beam-off period and the probability of observing it as a random event is extremely low. For the second SF, this probability is about $1 \%$.

In one of the decay chains observed at the $255-\mathrm{MeV}$ ${ }^{48} \mathrm{Ca}$ beam energy, the implantation of the ER in strip 1 was followed by an $\alpha$ particle with $E_{\alpha 1}=10.87 \mathrm{MeV}$ that switched the beam off (see Table II). During the 1-min pause, the next $\alpha$ particle was observed with $E_{\alpha 2}=10.23 \mathrm{MeV}$ in the same detector position, which prolonged the beam-off interval to 12 minutes. During this 12-min time interval only one $\alpha$ particle $\left(E_{\alpha}=8.92 \mathrm{MeV}\right)$ was registered in the side detector $4.69 \mathrm{~min}$ after the $\alpha$ particle with $E_{\alpha 2}=$ $10.23 \mathrm{MeV}$. No other $\alpha$ decays with $E_{\alpha} \geqslant 8 \mathrm{MeV}$ were observed in strip 1 during the beam-off interval, nor were any SF events. The missing SF event can be explained by a short decay time (during the 84 microseconds dead time of the electronic data acquisition system following detection of the $\alpha$ particle with $E_{\alpha 2}=10.23 \mathrm{MeV}, t_{\mathrm{SF}}<t_{\text {reg }}$ ). As for the longer time interval $\left(t_{\mathrm{SF}}>12 \mathrm{~min}\right)$, a SF decay with $E_{\mathrm{SF}}=185 \mathrm{MeV}$ and $\delta P_{\mathrm{ER}-\mathrm{SF}}=1.3 \mathrm{~mm}$ was observed in strip 1 only $17.31 \mathrm{~min}$ after the second $\alpha$ particle. The number of SF events that could be randomly detected after any beam-off interval in the same strip, position, and during a time interval of $17.3 \mathrm{~min}$ is about $\sim 0.2$. The energies of the first two $\alpha$ particles, as well as their lifetimes, agree with those previously measured for ${ }^{290} 116$ and ${ }^{286} 114$. Taking into account the uncertainty in the half-life of ${ }^{282} 112$ and the number of previously observed ${ }^{286} 114(\alpha) \rightarrow{ }^{282} 112$ (SF) decays, we can estimate the number of possibly lost SF events during the course of this experiment as 0.12-0.21. Therefore, we conclude that the SF decay of ${ }^{282} 112$ was missed, and suggest that it occurred during the electronic dead time $t_{\mathrm{SF}}<t_{\text {reg }}$.

In the experiment at $E_{\text {lab }}=249 \mathrm{MeV}$, we observed a long decay chain ER- $\alpha_{1}-\ldots-\alpha_{6}$-SF consisting of six consecutive $\alpha$ decays and terminated by a SF with a measured total fission-fragment energy of $240 \mathrm{MeV}$. The total decay time of all nuclei in this chain is about $0.4 \mathrm{~h}$. This sequence of decays belongs to the parent isotope ${ }^{291} 116$ produced via the 
$2 n$-evaporation channel of the ${ }^{245} \mathrm{Cm}+{ }^{48} \mathrm{Ca}$ reaction. The decay properties of ${ }^{291} 116$ were determined in a previous experiment [1]. In addition, the daughter isotope, ${ }^{287} 114$, was observed in two reactions, ${ }^{242} \mathrm{Pu}\left({ }^{48} \mathrm{Ca}, 3 n\right)$ and ${ }^{244} \mathrm{Pu}\left({ }^{48} \mathrm{Ca}, 5 n\right)$, and finally the granddaughter isotope, ${ }^{283} 112$, was produced in the ${ }^{238} \mathrm{U}\left({ }^{48} \mathrm{Ca}, 3 n\right)$ reaction $[1,2]$. The decay chains of isotopes ${ }^{291} 116,{ }^{287} 114$, and ${ }^{283} 112$ usually end in the spontaneous fission of ${ }^{279} \mathrm{Ds}\left(T_{1 / 2}=0.2 \mathrm{~s}\right)$. However, in three cases out of 26 observed decays (including this one), ${ }^{279}$ Ds underwent $\alpha$ decay $\left(b_{\alpha} \approx 10 \%\right)$, which was followed by the further $\alpha$ decay of ${ }^{275} \mathrm{Hs}$ and terminated in one case by the SF of ${ }^{271} \mathrm{Sg}\left(T_{1 / 2}=1.9 \mathrm{~min}\right)$ or in two other cases by another $\alpha$ decay and the $\mathrm{SF}$ of ${ }^{267} \mathrm{Rf}\left(T_{1 / 2}=1.3 \mathrm{~h}\right)$ [2]. One should note that assignment of an event with $E_{\alpha}=8.84 \mathrm{MeV}$ to ${ }^{271} \mathrm{Sg}$ in the decay chain observed in this experiment is somewhat tentative because its registration probability in a 1.6-min time interval as a random signal was about 0.25 . The long decay chain of the even-odd nucleus, ${ }^{291} 116$, is an interesting case of the transition from the region of heaviest nuclei $\left({ }^{291} 116\right.$ and ${ }^{287} 114$ ), whose stability is determined by the influence of possible spherical shell closures at $Z=114$ and $N=184$, to isotopes $\left({ }^{271} \mathrm{Sg}\right.$ or $\left.{ }^{267} \mathrm{Rf}\right)$ that are located near the deformed shells at $Z=108$ and $N=162$.

The production cross sections for the ${ }^{245} \mathrm{Cm}\left({ }^{48} \mathrm{Ca}\right.$, $2-4 n)^{289-291} 116$ reactions are shown in Fig. 2 together with the Bass reaction barrier [19] and the calculated excitation functions [23] for the $x n$-channels. The measured cross sections at excitation energy $E^{*}=37.9 \mathrm{MeV}$ are $\sigma_{2 n}=$ $0.7_{-0.6}^{+2.0} \mathrm{pb}$ and $\sigma_{3 n}=3.7_{-1.8}^{+3.6} \mathrm{pb}$. At the excitation energy $E^{*}=42.7 \mathrm{MeV}$, which corresponds to the maximum cross section of the $4 n$-evaporation channel of the ${ }^{245} \mathrm{Cm}+{ }^{48} \mathrm{Ca}$ reaction, four events of ${ }^{290} 116$ were observed, $\sigma_{3 n}=1.9_{-1.0}^{+2.1} \mathrm{pb}$ (see Table II), but no chain could be attributed to the decay of the neighboring even-odd nucleus ${ }^{289} 116$-the product of the $4 n$-evaporation. For this reaction channel we give an upper cross section limit of $\sigma_{4 n} \leqslant 1.0 \mathrm{pb}$ at $E_{\text {lab }}=255 \mathrm{MeV}$.

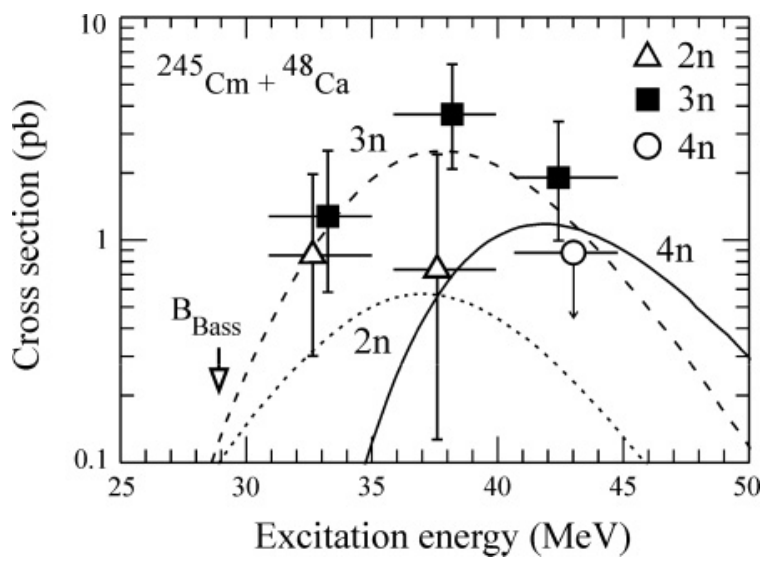

FIG. 2. Excitation functions for the $2 n, 3 n$, and $4 n$ evaporation channels from the complete-fusion reaction ${ }^{245} \mathrm{Cm}+{ }^{48} \mathrm{Ca}$. The Bass barrier [19] is shown by an open arrow. Lines show the results of theoretical predictions [23]. Error bars correspond to statistical uncertainties.

\section{B. Experiments with a ${ }^{249} \mathrm{Cf}$ target}

From the results of the experiments with the ${ }^{245} \mathrm{Cm}$ target, we determined the optimum conditions for an experiment aimed at the synthesis of element 118 in the ${ }^{249} \mathrm{Cf}+{ }^{48} \mathrm{Ca}$ reaction. In this reaction, one could not expect a noticeable yield of the $4 n$-evaporation products. The two- or threeneutron evaporation channels, leading to ${ }^{295} 118$ and ${ }^{294} 118$, respectively, look most probable. The maximum cross section, as in the ${ }^{245} \mathrm{Cm}+{ }^{48} \mathrm{Ca}$ reaction, is expected for the $3 n$ channel $\left({ }^{294} 118\right)$ at an excitation energy of the compound nucleus of about $35 \mathrm{MeV}$. In the present work, the energy of ${ }^{48} \mathrm{Ca}$ ions was increased by $6 \mathrm{MeV}$ as compared with the first experiment that was run at $E_{\mathrm{lab}}=245 \mathrm{MeV}$ in 2002; we expected that this would result in an increase in the yield of ${ }^{294} 118$ by a factor of 2-4. In the present experiment, the beam energy was $251 \mathrm{MeV}$, which corresponds to the excitation energy of 32.136.6 MeV of the compound nucleus, ${ }^{297} 118$ (see Table I).

The ${ }^{249} \mathrm{Cf}$ target was irradiated by ${ }^{48} \mathrm{Ca}$ ions for $1080 \mathrm{~h}$, with a total accumulated beam dose of $1.6 \times 10^{19}$ particles. During the irradiation, 3790 beam stops occurred, with the beam off for a total of $64.5 \mathrm{~h}$.

The spectrum of $\alpha$-like signals detected during the course of the ${ }^{249} \mathrm{Cf}+{ }^{48} \mathrm{Ca}$ experiment is shown in Fig. 3(a) with beam on the target and during beam-off periods. In beam-off intervals, over the course of the whole experiment, eight signals were detected in the $10 \leqslant E_{\alpha} \leqslant 11 \mathrm{MeV}$ energy interval corresponding to the $\alpha$ decays of daughters of $Z=$ 118 isotopes; two of them, as will be shown below, belong to the decays of ${ }^{286} 114$ and ${ }^{290} 116$. The spectrum of fission-like signals is shown in Fig. 3(b); 178 events occurred in the energy range expected for the SF of heavy nuclei. Compared with the ${ }^{245} \mathrm{Cm}+{ }^{48} \mathrm{Ca}$ reaction, the yield of fission events increased by about an order of magnitude. But even with such a fission-counting rate $\left(\approx 5 \times 10^{-5} / \mathrm{s}\right.$ over the total sensitive area of detector, and $\approx 5 \times 10^{-7} / \mathrm{s}$ within a $\Delta y=3 \mathrm{~mm}$ position window) the probability of random $\alpha$-SF correlations (within $\Delta t=1 \mathrm{~s})$ with beam-off $\alpha$ particles $\left(10 \leqslant E_{\alpha} \leqslant\right.$ $11 \mathrm{MeV})$ is negligible $\left(\sim 10^{-30}\right)$.

In the ${ }^{249} \mathrm{Cf}+{ }^{48} \mathrm{Ca}$ experiment, with a beam energy of $E_{\text {lab }}=251 \mathrm{MeV}$, two correlated decay chains were detected (see Table II). Also given in Table II is the decay chain observed during the prior experiment at a beam energy of $E_{\text {lab }}=$ $245 \mathrm{MeV}$ [18]. The decay patterns and characteristics of all three events coincide within the accuracy of energy measurement and statistical fluctuations of the decay times in the observed chains. Indeed, the implantation of the recoil nucleus in the detector with an expected energy of $E_{\mathrm{ER}}=7-16 \mathrm{MeV}$ was followed in an average time interval of $\bar{t}_{1} \approx 1.3 \mathrm{~ms}$ by emission of an $\alpha$ particle with $E_{\alpha}=11.65 \mathrm{MeV}$. In $\bar{t}_{2} \approx$ $14 \mathrm{~ms}$, the first decay was followed by another $\alpha$ decay, with $E_{\alpha}=10.80 \mathrm{MeV}$, and then, in $\bar{t}_{3} \approx 0.23 \mathrm{~s}$ by the decay of the granddaughter nuclide. In two of the chains, the granddaughter nucleus undergoes spontaneous fission; for one of them, both fission fragments were detected $E_{F 1}+E_{F 2}=207 \mathrm{MeV}$ $(\mathrm{TKE} \approx 230 \mathrm{MeV})$. In the third chain, the terminating spontaneous fission $\left(E_{F 1}+E_{F 2}=202 \mathrm{MeV}\right)$ was detected $2.7 \mathrm{~ms}$ after the emission of a third $\alpha$ particle with $E_{\alpha}=$ 10.16 MeV. 

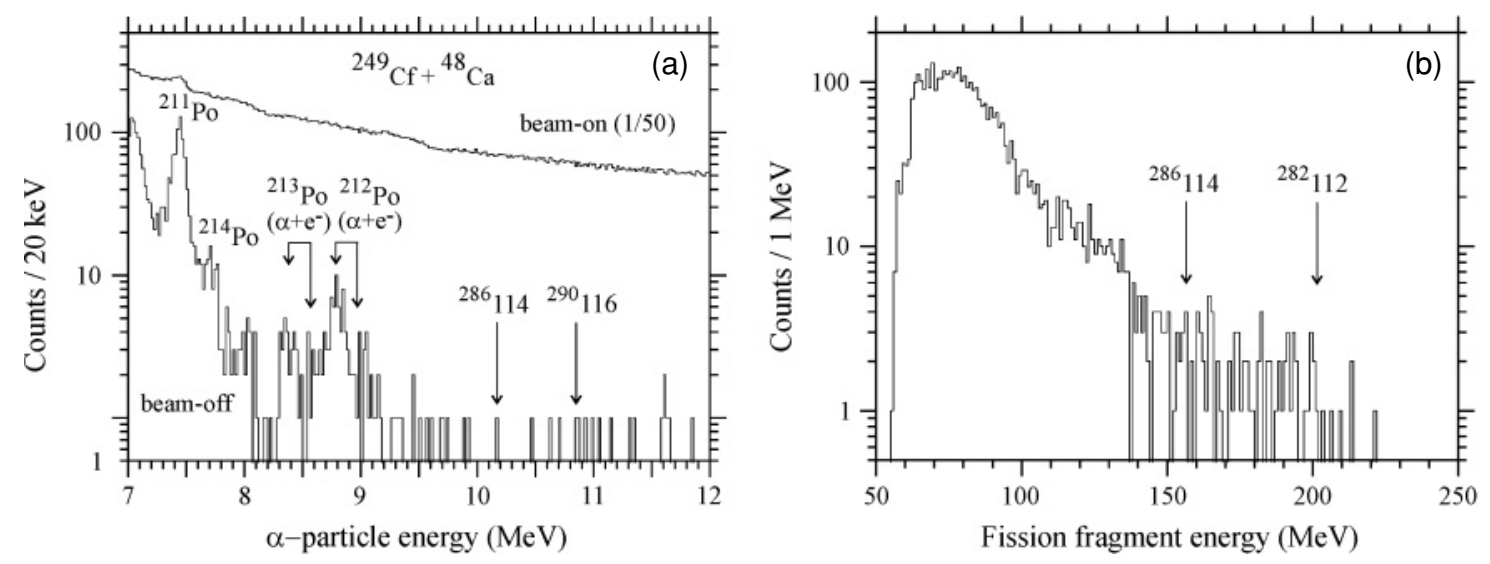

FIG. 3. The same as in Fig. 1 for the ${ }^{249} \mathrm{Cf}+{ }^{48} \mathrm{Ca}$ reaction.

As a whole, the positions measured in the strip detector support a correlation of the events within the detected chains (see Table II). The 6.2-mm position deviation observed for the $\alpha$ particle with $E_{\alpha}=10.71 \mathrm{MeV}$ is permissible in view of the low amount of energy (1.41 MeV) deposited in the focal-plane detector, as illustrated by the dependence shown in Ref. [18], Fig. 4. In one case, the position of the SF event deviates from the recoil by $2.5 \mathrm{~mm}$. However, the position of this event, observed during a beam-off time interval, deviates from preceding $\alpha$ particles by $1.8 \mathrm{~mm}$, which is within the position resolution for strip 7 . In any case, these deviations have no effect upon the final conclusions; the probabilities of random correlation events, constructed from the counting rate of beam-on $\alpha$ particles with $E_{\alpha}=10.5-$ $12 \mathrm{MeV}$ during $5 \mathrm{~ms}$, or beam-off $\alpha$ particles $\left(E_{\alpha}=10\right.$ $11 \mathrm{MeV}$ ) or fission fragments within 1-s time intervals, are negligible $\left(5 \times 10^{-4}, 3 \times 10^{-5}\right.$, and $5 \times 10^{-5}$, respectively) even without taking the position signals into account. Using these values one can show that the number of random ER$\alpha_{\text {beam-stop }}-\alpha_{\text {beam-off }}-\mathrm{SF}$ sequences of events detected within time interval of $1 \mathrm{~s}$ is about $6 \times 10^{-6}$. Moreover, using the Monte Carlo random probability (MCRP) method [24], a total of more than $5 \times 10^{8}$ random artificial events $\mathrm{SF}_{\mathrm{ar}}$ were inserted into the data to determine conservative probabilities that the events comprising the decay chains observed in both sets of experiments are really due to random coincidences. The following conditions were met to arrive at the probabilities calculated: $E_{\mathrm{ER}}=7-15 \mathrm{MeV}, \alpha$-energy gates of $\pm 0.5 \mathrm{MeV}$, position window $= \pm 2 \mathrm{~mm}$, and time gates of 8 times the actual time differences, without any Geiger-Nuttall semi-empirical relationship requirements. The decay chains consisting of any ER- $\alpha-\mathrm{SF}_{\mathrm{ar}}$ random events for the element 118 experiment or ER- $\alpha_{1}-\alpha_{2}-\alpha_{3}-\mathrm{SF}_{\mathrm{ar}}$ for the element 116 experiment would have a probability of being comprised of random events of $4 \times 10^{-8} / \mathrm{SF}_{\mathrm{ar}}$ and $2 \times 10^{-8} / \mathrm{SF}_{\mathrm{ar}}$, respectively. Taking into account the total number of SF events registered in the reactions with ${ }^{249} \mathrm{Cf}$ or ${ }^{245} \mathrm{Cm}$ (178 or 24 , see above), the number of random ER- $\alpha$-SF or ER- $\alpha_{1}-\alpha_{2}-\alpha_{3}-\mathrm{SF}$ chains of $7 \times 10^{-6}$ or $5 \times 10^{-7}$ might be expected to be observed in corresponding experiments. The MCRP method has been used for every recent ${ }^{48} \mathrm{Ca}+$ actinide experiment and has consistently indicated that the probability to observe a given decay sequence randomly is small $\left(<10^{-5}\right)$.

The average decay characteristics of the new nuclei observed in the ${ }^{249} \mathrm{Cf}+{ }^{48} \mathrm{Ca}$ reaction are shown in the left-hand part of Fig. 4. The right-hand part shows the decay chains of the two isotopes of element 116 previously produced in the ${ }^{245} \mathrm{Cm}+{ }^{48} \mathrm{Ca}$ experiment. From the comparison of the decay properties of the nuclei synthesized in the two experiments with targets of ${ }^{249} \mathrm{Cf}$ and ${ }^{245} \mathrm{Cm}$, it follows that in the ${ }^{249} \mathrm{Cf}+{ }^{48} \mathrm{Ca}$ reaction an isotope of the new element with $Z=118$ and $A=294$ was observed. It undergoes $\alpha$ decay leading to the daughter nucleus ${ }^{290} 116$, which was synthesized in the ${ }^{245} \mathrm{Cm}\left({ }^{48} \mathrm{Ca}, 3 n\right){ }^{290} 116$ reaction. Indeed, the $\alpha$-decay energies and lifetimes of the daughters of ${ }^{294} 118$ coincide, within experimental uncertainties, with those determined for the nuclei belonging to the ${ }^{290} 116(\alpha) \rightarrow{ }^{286} 114(\mathrm{SF} / \alpha) \rightarrow$ ${ }^{282} 112$ (SF) decay chain. In two of the three cases, spontaneous fission is observed for the granddaughter nucleus, in good agreement with the properties of ${ }^{286} 114$, which are characterized by $\alpha$ decay and SF with almost equal probabilities. Finally, in the long ER- $\alpha_{1}-\alpha_{2}-\alpha_{3}$-SF chain detected in the ${ }^{249} \mathrm{Cf}+{ }^{48} \mathrm{Ca}$ reaction, spontaneous fission occurs for the great-granddaughter nucleus, ${ }^{282} 112$. The most striking difference in the properties of the nuclei in the decay chains of ${ }^{294} 118$ and ${ }^{295} 118$ should show up for ${ }^{282} 112$ and ${ }^{283} 112$ (see Fig. 4). The observed spontaneous fission, with a short lifetime of $t_{\mathrm{SF}}=2.7 \mathrm{~ms}$, allows us to assign the SF to ${ }^{282} 112$ and the whole chain to the decay of the even-even ${ }^{294} 118$ isotope. Note that for the neighboring even-odd isotope ${ }^{283} 112$, different decay properties were measured $\left(E_{\alpha}=9.54 \mathrm{MeV}\right.$ and $\left.T_{\alpha}=3.8 \mathrm{~s}\right)$. It therefore belongs to the decay chain of ${ }^{295} 118$ (which was not observed).

Along with these three ER- $\alpha-\alpha-\mathrm{SF}$ or ER- $\alpha-\alpha-\alpha-\mathrm{SF}$ decay chains, a short ER-SF decay was registered in the first ${ }^{249} \mathrm{Cf}+{ }^{48} \mathrm{Ca}$ experiment [18]. Because its decay time $\left(t_{\mathrm{SF}}=\right.$ $3.16 \mathrm{~ms}$ ) was close to that of the first $\alpha$ transition with $E_{\alpha}=11.65 \mathrm{MeV}$ in the other decay chains, and the fission fragments had high total kinetic energy $\left(E_{\mathrm{SF}}=223 \mathrm{MeV}\right)$, this SF decay could be assigned to the direct spontaneous fission of ${ }^{294} 118$. However, in the second experiment no short 


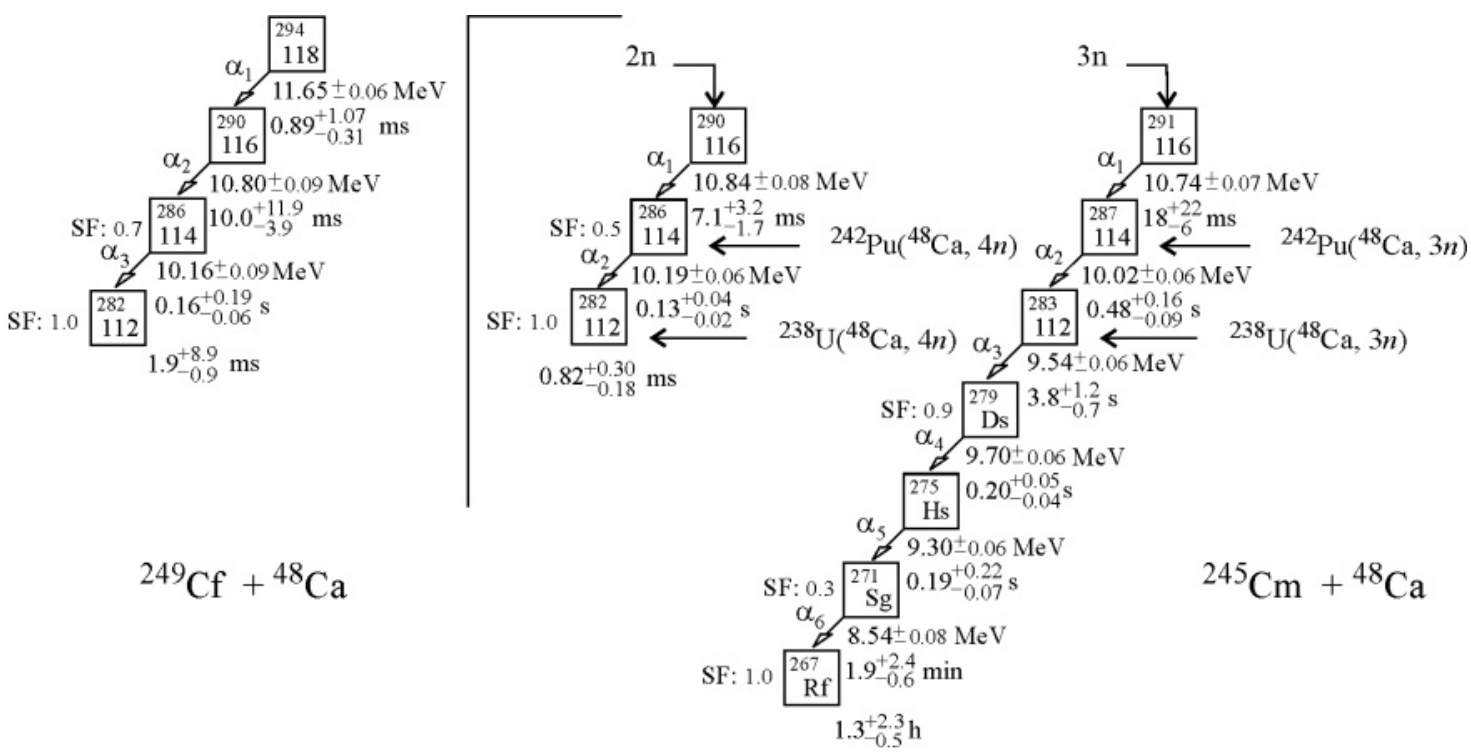

FIG. 4. Time sequences in the decay chains of ${ }^{294} 118$ (left), ${ }^{290} 116$ (middle), and ${ }^{291} 116$ (right) observed in the ${ }^{249} \mathrm{Cf}+{ }^{48} \mathrm{Ca}$ and ${ }^{245} \mathrm{Cm}+{ }^{48} \mathrm{Ca}$ reactions. The nuclei observed in cross bombardments of ${ }^{242} \mathrm{Pu}$ and ${ }^{238} \mathrm{U}$ by ${ }^{48} \mathrm{Ca}$ are shown by arrows. The average measured $\alpha$-particle energies, half-lives, and SF branching ratios of the observed nuclei are shown separately for three decay chains of ${ }^{294} 118$ and all nuclei in the chains originating from parent isotopes ${ }^{290} 116$ and ${ }^{291} 116$.

ER-SF events have been found. So, we can conclude that $\alpha$ decay is the prevalent decay mode of ${ }^{294} 118$ and set the SF branch upper limit of $b_{\mathrm{SF}} \leqslant 0.5$.

In Table III, the values of the half-lives, $\alpha$-decay energies and fission TKE values of the isotopes listed in Table II are averaged together with the data obtained for the same isotopes in our previous experiments, which were summarized earlier (Table IV of Ref. [2]).

The ${ }^{294} 118$ isotope was produced via the $3 n$-evaporation channel of the ${ }^{249} \mathrm{Cf}+{ }^{48} \mathrm{Ca}$ complete fusion reaction with a cross section of $0.5_{-0.3}^{+1.6} \mathrm{pb}$ at $E^{*}=32.1-36.6 \mathrm{MeV}$. An increase of the ${ }^{48} \mathrm{Ca}$ energy from $245 \mathrm{MeV}\left(\sigma_{3 n}=0.3_{-0.27}^{+1.0} \mathrm{pb}\right)$ to $251 \mathrm{MeV}$ resulted in an increase of the production of element 118 nuclei by a factor of about two. This was expected for the $3 n$-evaporation channel of the ${ }^{249} \mathrm{Cf}+{ }^{48} \mathrm{Ca}$ reaction because similar behavior was observed in the ${ }^{245} \mathrm{Cm}\left({ }^{48} \mathrm{Ca}, 3 n\right){ }^{290} 116$ reaction (reported in this work) as well as in other reactions with ${ }^{238} \mathrm{U},{ }^{242,244} \mathrm{Pu}$, and ${ }^{248} \mathrm{Cm}$ target nuclei [1,2]. In two experiments with the ${ }^{249} \mathrm{Cf}$ target in the excitation energy range $E^{*}=26.6-36.6 \mathrm{MeV}$, no decay chains of the neighboring ${ }^{295} 118$ isotope, the product of the ${ }^{249} \mathrm{Cf}\left({ }^{48} \mathrm{Ca}, 2 n\right){ }^{295} 118$ reaction, were observed $\left(\sigma_{2 n} \leqslant 0.9 \mathrm{pb}\right.$ at $\left.251-\mathrm{MeV}^{48} \mathrm{Ca}\right)$. The lower yield of the $2 n$ channel is in agreement with the data obtained with the ${ }^{245} \mathrm{Cm}$ target in the energy range $E^{*}=30.9$ 44.8 $\mathrm{MeV}$, where the cross section ratio for the production of the isotopes of element 116 was measured to be $\sigma_{2 n} / \sigma_{3 n} \approx$ $1 / 4$.

Experimental $Q_{\alpha}(\exp )$ values measured for the even-even nuclei ${ }^{294} 118,{ }^{290} 116$, and ${ }^{286} 114$ can be directly compared with those calculated in various microscopic nuclear models, $Q_{\alpha}$ (th). In comparing experiment with theory, we limit ourselves to two recent calculations with the MM model [4,5], five calculations with the HFB model (with different Skyrme or Gogny forces) [6-10] and two RMF calculations [11,12]. On average, the $\Delta Q_{\alpha}=Q_{\alpha}(\exp )-Q_{\alpha}($ th $)$ difference varies within $1 \mathrm{MeV}$ (see Fig. 5). The best agreement is observed with the MM calculations, especially with the version of [5] $\left(\Delta Q_{\alpha} \leqslant 0.2 \mathrm{MeV}\right)$.

Another aspect of proton shell effects is related to the production cross sections for the isotopes 290,291 116 and ${ }^{294} 118$. Detailed measurements of the excitation functions of $x n$-evaporation channels of the ${ }^{233,238} \mathrm{U},{ }^{242,244} \mathrm{Pu},{ }^{243} \mathrm{Am}$,

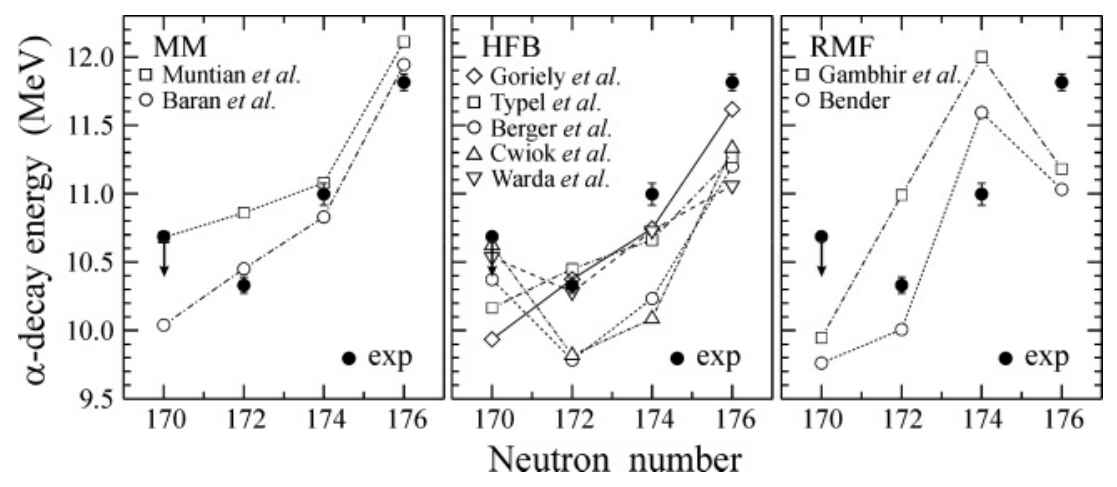

044602-7
FIG. 5. $\alpha$-decay energy $v s$. neutron number for isotopes in the decay chain originating from ${ }^{294} 118$. Solid circles show the experimental data. The value shown for ${ }^{282} 112$ is an upper limit, since this nuclide has not been observed to decay by $\alpha$-emission. Open symbols connected by lines show the theoretical $Q_{\alpha}$ values calculated for the same isotopes in the MM (squares [4], circles [5]), HFB (diamonds [6], squares [7], circles [8], triangles up [9], triangles down [10]) and RMF (squares [11], circles [12]) models. 
TABLE III. Decay properties of nuclei.

\begin{tabular}{|c|c|c|c|c|c|c|c|c|}
\hline$Z$ & $A$ & No. observed ${ }^{\mathrm{a}}$ & $\begin{array}{l}\text { Decay mode, } \\
\text { branch }(\%)^{\mathrm{b}}\end{array}$ & Half-life ${ }^{c}$ & $\begin{array}{l}\text { Expected } \\
\text { half-life }^{d}\end{array}$ & $\begin{array}{c}E_{\alpha}(\mathrm{MeV}) \\
\text { TKE }(\mathrm{MeV})\end{array}$ & $Q_{\alpha}(\mathrm{MeV})$ & Ref. \\
\hline \multirow[t]{2}{*}{116} & 291 & $3(3 / 3)$ & $\alpha$ & $18_{-6}^{+22} \mathrm{~ms}$ & $20 \mathrm{~ms}$ & $10.74 \pm 0.07$ & $10.89 \pm 0.07$ & this work, [1] \\
\hline & 290 & $10(10 / 10)$ & $\alpha$ & $7.1_{-1.7}^{+3.2} \mathrm{~ms}$ & $10 \mathrm{~ms}$ & $10.84 \pm 0.08$ & $11.00 \pm 0.08$ & this work, [1] \\
\hline 114 & 286 & $24(19 / 12 / 7)$ & $\alpha: 50 \mathrm{SF}: 50$ & $0.13_{-0.02}^{+0.04} \mathrm{~s}$ & $0.2 \mathrm{~s}$ & $\begin{array}{c}10.19 \pm 0.06 \\
222^{\mathrm{e}}\end{array}$ & $10.33 \pm 0.06$ & this work, $[1,2]$ \\
\hline 112 & 283 & $22(19 / 19)$ & $\alpha: 100 \mathrm{SF}: \leqslant 10$ & $3.8_{-0.7}^{+1.2} \mathrm{~s}$ & $3 \mathrm{~s}$ & $9.54 \pm 0.06$ & $9.67 \pm 0.06$ & this work, $[1,2]$ \\
\hline 108 & 275 & $3(3 / 3)$ & $\alpha$ & $0.19_{-0.07}^{+0.22} \mathrm{~s}$ & $0.8 \mathrm{~s}$ & $9.30 \pm 0.06$ & $9.44 \pm 0.06$ & this work, [2] \\
\hline 106 & 271 & $3(3 / 2 / 1)$ & $\alpha: 70 \mathrm{SF}: 30$ & $1.9_{-0.6}^{+2.4} \mathrm{~min}$ & $0.7 \mathrm{~min}$ & $\begin{array}{c}8.54 \pm 0.08 \\
248^{\mathrm{e}}\end{array}$ & $8.67 \pm 0.08$ & this work, [2] \\
\hline 104 & 267 & $2(2 /-/ 1)$ & $\mathrm{SF}$ & $1.3_{-0.5}^{+2.3} \mathrm{~h}$ & & $260^{\mathrm{e}}$ & $\leqslant 8.21$ & this work, [2] \\
\hline
\end{tabular}

${ }^{a}$ Number of observed decays and number of events used for calculations of half-lives / $\alpha$-particle energies / TKE, respectively.

${ }^{\mathrm{b}}$ Branching ratio is not shown if only one decay mode was observed.

${ }^{\mathrm{c}}$ Error bars correspond to $68 \%$-confidence level.

${ }^{\mathrm{d}}$ Half-lives calculated using the Viola-Seaborg formula (see text) for $\alpha$-decay energies, given in the next column.

${ }^{\mathrm{e}} \mathrm{TKE}$ obtained as the mean value of two registered fission fragments sum energies $E_{\mathrm{TOT}}( \pm 5 \mathrm{MeV})+20 \mathrm{MeV}$.

and ${ }^{245,248} \mathrm{Cm}+{ }^{48} \mathrm{Ca}[1-3]$ reactions reveal an increase in the fusion-evaporation cross sections $\sigma_{\mathrm{ER}}=\Sigma \sigma_{x n}$ with an increase in the neutron number of the compound nucleus. The growth of $\sigma_{\mathrm{ER}} v s . N$ is explained by an increased survivability of the compound nuclei that is associated with an increasing height of the fission barrier [2,23]. Indeed, according to the MM [5,25] and self-consistent HFB or RMF $[10,26,27]$ calculations, the fission barrier height of nuclei with $Z \geqslant 112$ increases considerably with increasing $N$ as one approaches the neutron shell $N=184$. However, in the MM approach, the fission barrier heights of nuclei with $Z>116$ decrease because they deviate from the magic proton number $Z_{\text {shell }}=114$. Qualitatively, this is in agreement with the cross sections measured in this work for ${ }^{290} 116\left(N_{\mathrm{CN}}=177\right)$ and ${ }^{294} 118\left(N_{\mathrm{CN}}=179\right)$ nuclei produced in the $3 n$-evaporation channels of the ${ }^{245} \mathrm{Cm}+{ }^{48} \mathrm{Ca}$ and ${ }^{249} \mathrm{Cf}+{ }^{48} \mathrm{Ca}$ reactions, respectively. In contrast, the self-consistent models predict an increase of the fission barrier for nuclei with $Z>116$ up to $Z=122-124$. Accordingly, the expected cross sections for producing compound nuclei with $Z=118$, or at least their survival probabilities, should be larger than those associated with the production of isotopes of elements 114 and 116 $\left(N_{\mathrm{CN}}=176-180\right)$ measured in the ${ }^{242,244} \mathrm{Pu},{ }^{245,248} \mathrm{Cm}+{ }^{48} \mathrm{Ca}$ reactions. This was not observed in our experiments; on the contrary, the yield of ${ }^{294} 118$ nuclei is about one order of magnitude lower than that of ${ }^{290} 116$ nuclei, as well as other previously observed isotopes of elements 114 and 116. In terms of shell structure, such a difference in the magnitude of the cross sections could indicate the influence of a proton shell at $Z<118$. Unfortunately, the quantitative analysis of all the factors determining final production cross sections of the superheavy nuclei is rather complicated. As a consequence, empirical evaluation of the fission barriers from the data on fusion-fission reactions and ER-production cross sections needs far more investigation.

\section{CONCLUSIONS}

A new element with atomic number 118 was synthesized for the first time in the ${ }^{249} \mathrm{Cf}+{ }^{48} \mathrm{Ca}$ reaction. Atomic and mass numbers of the isotope of element 118 were determined from the measured excitation functions and decay characteristics of the daughter nuclei produced in cross-bombardments. The isotope of the element 116 daughter was studied in the ${ }^{245} \mathrm{Cm}\left({ }^{48} \mathrm{Ca}, 3 n\right){ }^{290} 116$ reaction and the isotope of the element 114 granddaughter was studied in the ${ }^{242} \mathrm{Pu}\left({ }^{48} \mathrm{Ca}, 4 n\right){ }^{286} 114$ reaction. The even-even ${ }^{294} 118$ nuclide undergoes consecutive decays, $\alpha_{1} \rightarrow \alpha_{2} \rightarrow \mathrm{SF} / \alpha_{3} \rightarrow \mathrm{SF}$. The decay chains are terminated by the spontaneous fission of the granddaughter or great-granddaughter nuclei, ${ }^{286} 114$ or ${ }^{282} 112$, respectively. The $\alpha$-decay energy of ${ }^{294} 118$ is $Q_{\alpha}=11.81 \pm 0.06 \mathrm{MeV}$ and the half-life is $T_{\alpha}=0.89_{-0.31}^{+1.07} \mathrm{~ms}$; these decay properties are in agreement with that one would expect for the ground-to-ground state $\alpha$ transition of an element 118 isotope 
from the systematic behavior of $T_{\alpha}$ vs. $Q_{\alpha}$ for even-even isotopes [2,18]. The measured $Q_{\alpha}, T_{\alpha}$ and $T_{\mathrm{SF}}$ values of the ${ }^{294} 118,{ }^{290} 116,{ }^{286} 114$, and ${ }^{282} 112$ nuclei agree well with the properties of other previously synthesized isotopes [1-3] and with the theoretically predicted properties of the superheavy nuclei in the region of $Z=110-118$ and $N=169-177$.

The nuclei of element 118 are produced in the $3 n$ evaporation channel of the ${ }^{249} \mathrm{Cf}+{ }^{48} \mathrm{Ca}$ complete fusion reaction with a cross section of $0.5_{-0.3}^{+1.6} \mathrm{pb}$ at $E^{*}=32.1-36.6 \mathrm{MeV}$. The magnitude of the cross section, compared with those obtained from other reactions with ${ }^{48} \mathrm{Ca}$ projectiles, could indicate the influence of a closed proton shell at $Z<118$.

\section{ACKNOWLEDGMENTS}

We are grateful to the JINR Directorate for the support we received during all stages of the experiment. We would like to express our gratitude to the personnel of the U400 cyclotron and the associates of the ion-source group for obtaining intense ${ }^{48}$ Ca beams; G. V. Buklanov, A. N. Shamanin, B. A. Shestakov, Yu. G. Toporov, V. M. Radchenko, and M. A. Ryabinin for their help in preparing the ${ }^{249} \mathrm{Cf}$ and ${ }^{245} \mathrm{Cm}$ targets; V. I. Krashonkin, A. M. Zubareva, G. N. Ivanov, and V. B. Galinskiy for their help in performing the experiment. This work was performed with the support of the Russian Ministry of Atomic Energy and grant of RFBR No. 04-02-17186. The ${ }^{249} \mathrm{Cf}$ target material was provided by the U.S. DOE through ORNL and partially by RIAR, Dimitrovgrad. The ${ }^{245} \mathrm{Cm}$ target material was provided by RFNC-VNIIEF, Sarov, Russia. Much of the support for the LLNL authors was provided through the U.S. DOE under Contract No. W-7405-Eng-48. These studies were performed in the framework of the Russian Federation/U.S. Joint Coordinating Committee for Research on Fundamental Properties of Matter.
[1] Yu. Ts. Oganessian et al., Phys. Rev. C 69, 054607 (2004).

[2] Yu. Ts. Oganessian et al., Phys. Rev. C 70, 064609 (2004); [http://www.jinr.ru/publish/Preprints/2004/160(E72004-160).pdf] (unpublished).

[3] Yu. Ts. Oganessian et al., Phys. Rev. C 72, 034611 (2005); 69, 021601(R) (2004).

[4] I. Muntian, Z. Patyk, and A. Sobiczewski, Phys. At. Nucl. 66, 1015 (2003).

[5] A. Baran, Z. Łojewski, K. Sieja, and M. Kowal, Phys. Rev. C 72, 044310 (2005); A. Baran, Z. Łojewski, and K. Sieja, Acta Phys. Pol. B 36, 1369 (2005).

[6] S. Goriely, M. Samyn, P.-H. Heenen, J. M. Pearson, and F. Tondeur, Phys. Rev. C 66, 024326 (2002).

[7] S. Typel and B. A. Brown, Phys. Rev. C 67, 034313 (2003).

[8] J. F. Berger, D. Hirata, and M. Girod, Acta Phys. Pol. B 34, 1909 (2003).

[9] S. Ćwiok, W. Nazarewicz, and P. H. Heenen, Phys. Rev. Lett. 83, 1108 (1999); S. Ćwiok, P.-H. Heenen, and W. Nazarewicz, Nature (London) 433, 705 (2005).

[10] M. Warda, J. L. Egido, and L. M. Robledo, in Proceedings of the 12th Nuclear Physics Workshop "Marie and Pierre Curie," Kazimierz Dolny, Poland, 21-25, Sep. 2005, edited by A. Baran, J. Bartel, and K. Pomorski; [Int. J. Mod. Phys. E 15, 504 (2006)].

[11] Y. K. Gambhir, A. Bhagwat, and M. Gupta, Ann. Phys. (NY) 320, 429 (2005); Phys. Rev. C 71, 037301 (2005).

[12] M. Bender, Phys. Rev. C 61, 031302(R) (2000).

[13] R. Smolańczuk, Phys. Rev. C 59, 2634 (1999); 63, 044607 (2001).

[14] S. Hofmann and G. Münzenberg, Rev. Mod. Phys. 72, 733 (2000).

[15] K. Moritomo et al., in Proceedings of the Tours Symposium on Nuclear Physics IV, Tours, France, 2000 (American Institute of Physics, New York, 2001), p. 354.
[16] C. Stodel et al., in Ref. [15], p. 344.

[17] K. E. Gregorich et al., Eur. Phys. J. A 18, 633 (2003).

[18] Yu. Ts. Oganessian et al., JINR Communication D7-2002287 (2002) [http://www.jinr.ru/publish/Preprints/2002/287(D72002-287)e.pdf] (unpublished); Lawrence Livermore National Laboratory Report, UCRL-ID-151619 (2003) (unpublished); Nucl. Phys. A734, 109 (2004).

[19] R. Bass, in Proceedings of the Symposium on Deep Inelastic and Fusion Reactions with Heavy Ions, West Berlin, 1979, edited by W. von Oertzen, Lecture Notes in Physics (Springer-Verlag, Berlin, 1980), Vol. 117, p. 281.

[20] Yu. Ts. Oganessian et al., in Proceedings of the Fourth International Conference on Dynamical Aspects of Nuclear Fission, Častá-Papiernička, Slovak Republic, 1998 (World Scientific, Singapore, 2000), p. 334; K. Subotic et al., Nucl. Instrum. Methods Phys. Res. A 481, 71 (2002).

[21] W. D. Myers and W. J. Swiatecki, Nucl. Phys. A601, 141 (1996).

[22] F. Hubert, R. Bimbot, and H. Gauvin, At. Data Nucl. Data Tables 46, 1 (1990); L. C. Northcliffe and R. F. Schilling, Nucl. Data Tables A 7, 233 (1970).

[23] V. I. Zagrebaev, in Proceedings of the Tours Symposium on Nuclear Physics V, Tours, France, 2003 (American Institute of Physics, New York, 2004), p. 31; Nucl. Phys. A734, 164 (2004).

[24] N. J. Stoyer et al., Nucl. Instrum. Methods Phys. Res. A 455, 433 (2000).

[25] I. Muntian, Z. Patyk, and A. Sobiczewski, Acta Phys. Pol. B 34, 2141 (2003).

[26] T. Bürvenich, M. Bender, J. A. Maruhn, and P.-G. Reinhard, Phys. Rev. C 69, 014307 (2004).

[27] M. Samyn, S. Goriely, and J. M. Pearson, Phys. Rev. C 72, 044316 (2005). 